\title{
On Vehicle routing Problem using Mixed Integer Non-Linear Programming
}

\author{
${ }^{1}$ Mamta Raipuriya, ${ }^{2}$ Richa Gupta, \\ ${ }^{1}$ Reasearch Scholar, ${ }^{2}$ Professor, Dept. of Mathematics, SRK Univ.,Bhopal, India. \\ mamtaraipuriya@gmail.com
}

\begin{abstract}
The family of (VRPs) has received remarkable attention in the field of combinatorial optimization after its introduction in the paper of Dantzig and Ramser. VRPs determine a set of vehicle routes in order to accomplish transportation requests at minimum cost. In this paper we develop a mixed-integer non-linear programming model for vrp and apply it in electric vehicle charging.
\end{abstract}

Keywords: Vehicle Routing Problems, mixed-integer non-linear programming, electric vehicle charging.

\section{INTRODUCTION}

A key element of many distribution systems is the routing and scheduling of vehicles through a set of nodes requiring service. The Vehicle Routing Problem (VRP) involves the design of a set of minimum-cost vehicle routes, originating and terminating at a central depot, for a fleet of vehicles that services a set of nodes with known demands. Each node is serviced exactly once and furthermore, all nodes must be assigned to vehicles without exceeding vehicle capacities (Bodinetal 1983).

Vehicle routing is defined by decisions, objectives and constraints. Fundamentally, the decisions of vehicle routing are to assign a group of nodes to $\operatorname{depot}(\mathrm{s})$ and to groups of drivers and vehicles, and to sequence and schedule their visits. The objective of vehicle routing is to provide a high level of customer service while keeping the operating and investment costs as low as possible.VRP consists of two sub-problems: the nodes grouping to clusters and finding the best tour for every cluster. Therefore, route is the total number of deliveries made by a single vehicle and tour is their sequence. The solution of these sub-problems results to the routes and tours that minimize the total transportation cost. VRP has received considerable attention over the last two decades. VRP has received considerable attention over the last two decades. Many efforts in the literature have been established to extend the basic VRP model to incorporate additional constraints or different objective functions.

VRP has many application in different areas. One of them is about electric vehicle. The increasing presence of Battery-Powered Vehicles(BPVs), such as Electric Vehicles (EVs) or mobile robots and sensors, has given rise to novel issues in classical network routing problems

This paper is organized as follows: In Section 2, we discuss about Vehicle Routing Problem and Mixed Integer Non-Linear Programming. In Section 3, we formulate the single EV routing problem as Mixed Integer Non-Linear Programming. In section 4, Conclusions and further research directions are outlined.

\section{VEHICLE ROUTING PROBLEM(VRP)}

The formulation of the TSP by Dantzig, Fulkerson and Johnson was extended to create the two index vehicle flow formulations for the VRP

Min $\quad \sum_{i \in V} \sum_{j \in V} c_{i j} x_{i j}$

Subject to

$$
\begin{aligned}
& \sum_{i \in V} x_{i j}=1, \forall j \in V \backslash\{0\} \\
& \sum_{j \in V} x_{i j}=1, \forall i \in V \backslash\{0\} \\
& \sum_{i \in V} x_{i 0}=\mathrm{K} \\
& \sum_{j \in V} x_{0 j}=\mathrm{K} \\
& \sum_{i \notin S} \sum_{j \in S} x_{i j} \geq r(S), \forall S \subseteq V \backslash\{0\}, S \neq \varnothing
\end{aligned}
$$

$$
x_{i j} \in\{0,1\}, \quad \forall i, j \in V
$$

In this formulation $c_{i j}$ represents the cost of going from node $\mathrm{i}$ to node $\mathrm{j}, x_{i j}$ is a binary variable that has value 1 if the arc going from $i$ to $j$ is considered as part of the solution and 0 otherwise. $\mathrm{K}$ is the number of available vehicles and $r(S)$ corresponds to the minimum number of vehicles needed to serve set $\mathrm{S}$. We are also assuming that 0 is the depot node.

Constraints 1 and 2 state that exactly one arc enters and exactly one leaves each vertex associated with a customer, respectively. Constraints 3 and 4 say that the number of 
vehicles leaving the depot is the same as the number entering. Constraints 5 are the capacity cut constraints, which impose that the routes must be connected and that the demand on each route must not exceed the vehicle capacity. Finally, constraints 6 are the integrality constraints.

\section{MIXED INTEGER NON-LINEAR Programming}

Mixed integer nonlinear programming (MINLP) refers to optimization problems with continuous and discrete variables and nonlinear functions in the objective function and/or the constraints. MINLPs arise in applications in a wide range of fields, including chemical engineering, finance, and manufacturing. The general form of a MINLP is

$$
\begin{array}{ll}
\text { Min } & f(x, y) \\
\text { s.t } & c_{i}(x, y)=0, \forall i \in E \\
& c_{i}(x, y) \leq 0, \forall i \in I \\
& x \in X, y \in Y \quad \text { integer }
\end{array}
$$

where each $c_{i}(x, y)$ is a mapping from $\mathrm{R}^{\mathrm{n}}$ to $\mathrm{R}$, and $\mathrm{E}$ and $\mathrm{I}$ are index sets for equality and inequality constraints, respectively. Typically, the functions $f$ and $c_{i}$ have some smoothness properties, i.e., once or twice continuously differentiable.

Formulation of Mixed Integer Non-Linear Programming in single vehicle routing for electric vehicle charging:

We assume, as in [1] and [2], that a network is defined as a directed graph $G=(N, A)$ with $N=\{1, \ldots, n\}$ and $|A|=m$. Node $i \in N \backslash\{n\}$ represents a charging station and $(i, j) \in A$ is an arc connecting node $\mathrm{i}$ to $\mathrm{j}$ (we assume for simplicity that all nodes have a charging capability, although this is not necessary). We also define $I(i)$ and $O(i)$ to be the set of start nodes (respectively, end nodes) of arcs that are incoming to (respectively, outgoing from) node i, that is, $I(i)=\{j \in N \mid(j . i) \in A$ and $O(i)=j \in$ $N \mid(i . j) \in A$..

First, we deal with a single-origin-single-destination vehicle routing problem in a network of inhomogeneous charging stations. Nodes 1 and $\mathrm{n}$ respectively are defined to be the origin and destination. For each $\operatorname{arc}(i, j) \in A$, there are two cost parameters: the required travelling time $T_{i j}$ and the energy consumption $e_{i j}$. Note that $T_{i j}>0$ (if nodes $\mathrm{i}$ and $\mathrm{j}$ are not connected, then $T_{i j}=\infty 1$ ), whereas $e_{i j}$ is allowed to be negative due to an EV's potential energy recuperation effect [4]. Letting the vehicle's charge capacity be B, we assume that $e_{i j}<\mathrm{B}$ for all $(i, j) \in A$. Since we are considering a single vehicle's behaviour, we assume that it will not affect the overall network's traffic state, therefore, $T_{i j}$ and $e_{i j}$ are fixed depending on given traffic conditions at the time the single-vehicle routing problem is solved.
Clearly, this cannot apply to the multi-vehicle case in the next section, where the decisions of multiple vehicle routes affect traffic conditions, thus influencing traveling times and energy consumption. Since the EV has limited battery energy, it may not be able to reach the destination without recharging. Thus, recharging amounts at charging nodes $i \in N$ are also decision variables.

The single vehicle's objective is to determine a path from 1 to $\mathrm{n}$, as well as recharging amounts, so as to minimize the total elapsed time to reach the destination. We formulate this as a Mixed Integer Nonlinear Programming (MINLP) problem:

$\min _{x_{i j} r_{i} i, j \in N} \quad \sum_{i-1}^{n} \sum_{j=1}^{n} T_{i j} x_{i j}+\sum_{i=1}^{n} \sum_{j=1}^{n} r_{i} g_{i} x_{i j}$

\section{Subject to}

$$
\begin{aligned}
& \sum_{j \in O(i)} x_{i j}-\sum_{j \in I(i)} x_{j i}=b_{i}, \text { for each } i \in N \\
& b_{1}=n, b_{n}=-1, b_{i}=0 \text { for } i \neq 1, n \\
& E_{j}=\sum_{i \in I(j)}\left(E_{i}+r_{i}-E_{i j}\right) x_{i j} \quad \text { for } j=2 \ldots \ldots n
\end{aligned}
$$

$0 \leq E_{i} \leq B, E_{1}$ given for each $i \in N$

$$
x_{i j} \in\{0,1\}, r_{i} \geq 0
$$

where $x_{i j} \in\{0,1\}, i, j \in N$ denotes the selection of arc $(i, j) . r_{i} \geq 0, i \in N \backslash\{n\}$ is for energy recharging amount at node i, $E_{i}$ represents the vehicle's residual battery energy at node $\mathrm{i}$, and $g_{i}$ is the charging time per unit of energy for charging node i. The constraints (2)-(3) stand for flow conservation, which implies that only one path starting from node i can be selected. Constraint (4) represents the EV's energy dynamics. Finally, (5) indicates that the vehicle cannot run out of energy before reaching a node or exceed a given capacity $B$.

\section{CONCLUSION AND FUTURE WORK}

In this paper we have discussed single vehivle routing problem of electic vehicle and formulated it as a mixed integer non-linear programming .Furthermore, starting with a MINLP formulation, we can derived it as a DP problem. For a single vehicle, this approach is very efficient and determines an optimal solution in seconds.

\section{REFERENCES}

[1] T. Wang, C. Cassandras, and S. Pourazarm, "Energyaware vehicle routing in networks with charging stations," in To appear in Proc. Of 2014 IFAC World CongressarXiv:1401.6478.

[2] S. Pourazarm and C. Cassandras, "Optimal routing of energy-aware vehicle in networks with inhomogeneous charging nodes," in Proc. Of 22nd IEEE Mediterranean Conference on Control and Automation, June 2014, pp. 674-679. 
[3] G. Laporte, "The vehicle routing problem: An overview of exact and approximate algorithms," European Journal of Operational Research vol. 59, 1992.

[4] A. Artmeier, J. Haselmayr, M. Leucker, and M. Sachenbacher, "The optimal routing problem in the context of battery-powered electric vehicles," in Workshop: CROCS at CPAIOR-10, 2nd International Workshop on Constraint Reasoning and Optimization for Computational Sustainability, Bologna, Italy, May 2010.

[5] M. Schneider, A. Stenger, and D. Goeke, "The electric vehicle routing problem with time windows and recharging stations," Tech Report, Dept. of Business Information Systems and Operations Research, University of Kaiserslautern, 2012.

[6] D. P. Bertsekas, Dynamic Programming and Optimal Control, 4th ed. Athena Scientific, 2012, vol. I.

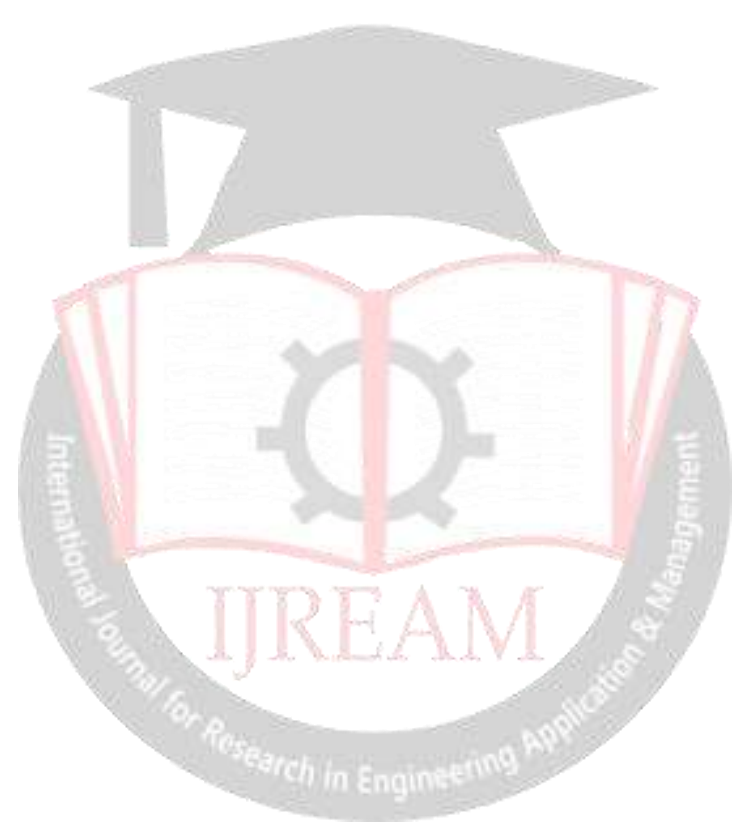

